\title{
LA LUTTE CONTRE LA STRONGYLOSE GASTRO-INTESTINALE EN ALGERIE
}

\author{
par JORE D'ARCES
}

Au cours de. sa réunion du 9 Juin 19k9, le Comité Algérien de l'Élevage - comité consultatif groupant éleveurs, techniciens $€$ t notabilités du monde scientifique nord-africain - soumit pour approbation au Gouverneur Général de l'Algérie la résolution suivante : "l'ampleur des ravages commis dans le cheptel ovin par les strongyloses pulmonaire et gastro-intestinale commande la mise en ouvre d'une lutte généralisée et immédiate contre les parasites, causes de ces affections. Les médicaments nécessaires à cette entreprise seront $\grave{a}$ mettre gratuitement $a$ la disposition des pasteurs musulmans qui en feront la demande, par l'intermédiaire des vétérinaires, inspecteurs du Service de l'Élevage ". .

Cette résolution fut immédiatement adoptée par le Gouverneur Général, en même temps qu'un important crédit destiné à l'achat de parasit: cides divers était mis à la disposition du Service de l'Élevage.

Poup mener à bien la tâche définie, il était indispensable d'établir un plan de travail, de marquer les étapes à parcourir et de prévoir les innombrablos obstacles qui allaient surgir en cours de ronte. Le problème posé avait les données suivantes :

$1^{\circ}$ Les pertes annuelles pour strongyloses oscillent entre 10 et $15 \%$ des moutons adultes et 30-35\% \% $\%$ agneaux. Dans certaines régions (Chellala-Aflou)

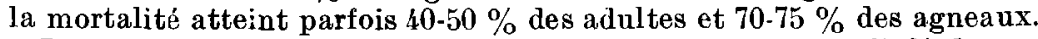

Le taux de morbidité est énorme. On peut estimer que $95 \%$ des moutons algériens sont porteurs de strongles.

$2^{\circ}$ Les éleveurs musulmans connaissent les strongyloses, mais pour eux il $n^{\prime} y$ a aucun traitement. A la rigueur, l'eau contenue dans les trous creusés au milieu de la steppe et provenant des premières pluies d'automne aurait une légère action curative, mais le souverain guérizseur est Dieu et on ne peut aller contre sa volonté.

$3^{\circ}$ Aussi curieux que cela puisio paraitre, l'inventaire méthodique de la faune parasitaire du cheptel ovin en Algérie n'a pas encore été dressé. Bien que des renseignements ${ }^{-p}$ précieux $\mathrm{p}$ uissent être tirés des travaux de G. Seurat sur les Nématodes, une analyse systématique des espèces parasites du mouton reste e core à faire. Nous̀ dirons ultérieurement où en sont les études entreprises aujourd'hui à ce sujet.

$4^{\circ}$ Le ou les vermicides préconisés devront être efficaces, à coup sûr. Le pasteur algérien, déjà difficile à convainore de l'utilité d'un médicament, ne doit pas connaître d'échec, ce serait perdre la confiance qu'il met dans son vétérinaire, son " toubib". Le ou les vermicides devront pouvoir être employós d'cmblée, sans que le troupeau ait à subir une préparation préalable (jeûne ou repos). Ils seront d'une administration facile pu:sque appelés à être utilisés, autant que possible, par les bergers eux-mêmes et d'un prix peu

Elev. et Méd. vét. des Pays tropicaux. - Oct.-Déc. 1948. 
élevé. Les résultats attendus devront être atteints sans a voir recours à des administrations répétées.

La recherche du vermicide idéal constituait donc le centre du problème.

Ce vermicide trouvé, il ne resterait plus qu'à organiser la propagande, le contrôle scientifique et la statistique.

Le Vermicide. : Après avoir passé en revue l'arsenal anthelminthique et étudié les propositions commerciales de diverses maisons productrices, l'Inspection Centrale du Service de l'Élevage pensa relenir pour commencer le sulfate de cuivre arsénié pour traiter les strongyloses gastro-intestinales et la solution éthérée d'huile pyréthrinée et créosotée pour les'strongyloses pulmonaires.

De nombreux vétérinaires algériens s'étaient déjà adressés à ses produits et s'en étaient déclarés sätisfaits, sans qu'il ait été toutefois possible d'exercer un contrôle parasitologique précis de leur action.

Les premières commandes allaient être passées, les premiers essais tentés quand, le 8 novembre 1942, les troupes alliées touchaient le sol algérien: L'Algérie, coupée de la Métropole, devait brusquement vivre sur ses propres ressources et sur ses réserves, les autorités britanniques et américaines consacrant leur shipping disponible à l'importation de produits pharmaceutiques plus urgents et plus indispensables que l'huile créosotée ou lesulfate de cuivre. C'ést dire qu'on balança' un temps entre l'idée de remettre à des jours meilleurs le traitement des ovins parasités et celle de l'entreprendre cô̂te que coûte, avec les moyens du bord. Allait-on recenser et bloquer nos avoirs en tétrachlorure de carbone, en extrait éthéré de' fougère mâle et 'en pyréthrines? Le Service-de l'Élevage eut alors l'occasion de prendre directement contact avec un organisme américain nouvellement installé à Alger — le NorthAfrica Economic Board (N.A.E.B.) - et de plaider la cause des moutons algériens privés de leurs médicaments les plus indispensables. Les arguments invoqués furent entiendus à Washington. Au moịs d'Août 1943, les États-Unis mettaient à la disposition de l'Algérie sept tonnes de phénothiazine.. En même temps, le N.A.E.B. transmettait au Service de l'Élevage une notice indiquant le mode d'emploi du produit et rédigé par M. BENjamin SchwarTz, zoologiste principal de la Division de Zoologie du Département de l'Agriẹuturo en U.S.A.

\section{Cette notice était ainsi conçue :}

“ La Phénothiazine peut êtré administrée en capsules (ce conditionnement eût parfaitement convenu dans le bled), dans la pâture ou sous forme de breuvage et dans tous les cas sous la surveillance d'un vétérinaire. ")

"Une solution satisfaisante pour l'absorption sous forme de breuvage peut être préparée en mélangeant parfailement et minulieusement 25 grammes de la drogue avec 15 cc. de mélasse et en y ajoutant de l'eau chaude' en quantité suffisante pour obtenir environ 45 cc.' ou 1,5 once.

"L'utilisation de la phénothiazine dans la pâture est plus prisée des éleveurs, car elle représente une méthode plus simple queles deux autres. Toutefois, il est désirable que seulement quelques bêtes par groupe soient ainsi traitées et ces bêtes doivent être suffisamment affamées pour 
cansommer la pâture médicamentée en quelques heures ou une journèe au plus.

« La formule suivante a été adoptée pour le traitement de bêtes par groupe de cinq :

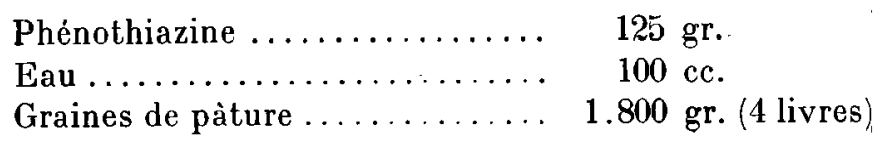

"Quand les animaux sont traités par groupes, il est nécessaire qu'ily ait la place voulue pour tous autour de l'auge, a fin qu'ils se nourrissent lous en même temps. Les bêtes débilitées devront faire l'objet d'une surveillance étroite afin d'être sûr qu'ils consomment une quantilé suffisante du mélunge médicamenté. De plus, ils doivent avoir de l'eau potable à tous les instants.

“ Cependant, étant donné que la phénothiazine est plutôt chère, son emploi est quelque peu limité."

Dans un pays où l'eau est rare et la mélasse absente, il devenait impossible de retenir de telles indications. Mais la phénothiazine était en magasin : il fallait la payer et l'utiliser.

Ce fut le travail d'une part, du laboratoire de Biologie générale et appliquée de la Faculté des Sciences d'Alger et, d'autre part, du Docteur-vétérinaire Visconrisi, inspecteur du Service de l'Élevage de l'annexe de Djelfa, de mettre au point l'utilisation de la phénothiazine en poudre dans le traitement des strongyloses gastro-intestinales du mouton. Dans une note, Maurice Rose, professeur de Biologie générale et appliquée à la Faculté des Sciences d'Alger, écrivait en conclusion de ses premières recherches :

“ La phénothiazine peut être adoptée pour traiter en grand les strongyloses gastro-intestinales du mouton. Elle doit donner des résultats appréciables.

"On peut l'administrer en poudre... Le médicament étant à peu près insoluble dans l'eau, il n'y a pas de raison de mettre les moutons à la diète alimentaire ou hydrique.

a... La dose à administrer doit être au minimum de $25 \mathrm{gr}$. pour avoir des effets durables... Deux cuillerées à bouche ordinaires valent environ 30 gr. de phénothiazine et constituent une dose excellente.

“ On pourrait, au moins au début, se contenter d'un seul traitement annuel, à donner dès le printemps, au moment oủ la maladie commence à apparaître dans les troupeaux.

Un second traitement en automne serait à préconiser, si des raisons d'ordre économique ne s'y opposent pas."

De son côté, V. Viscontini relevait les observations suivantes :

" 900 animaux, d'une part, et 300 environ de l'autre, ont subi un premier traitement à la phénothiazine. 
“ Ce traitement eut lieu le 5 Mars 1944 pour les 900 premiers. Je suivais ce troupeau depuis Mai 1943, époque des bains antigaleux. Malgré l'abondance des pacages du printemps passé, il était dêjà dans un état nettement moindre que ceux de la même région. Jé l'avais revu en Octobre 1943, au'cours d'une séance de vaccination anticlavéleuse. Là encore, l'état général était au-dessous de la moyenne, et le propriétaire, au cours de l'hiver, se plaignait d'une forte mortalité. C'est la raison qui m'a déterminé à choisir ces animaux pour expérimenter la phẻnothiazine. Parmi eux, un quart environ était cliniquement átteint de strongylose gastro-intestinale, mais aussi de strongylose pulmonaire: amaigrissement extrême, laine terne s'arrachant par endroit, yeux chassieux, conjonctives subanémiques, narines sales. Sur la demande du propriétaire, une cinquantaine de ces malades subirent, outre le traitement à la phénothiazine, l'injection intratrachéale d'huile pyréthrinée et créosotée en solution éthérée.

"Il est regrettable que les nécessités de la transhumance aient entraîné ce troupeau hors de ma portée. Jen'ai pu ainsi constater, par moi-même, les résultats du traitement.

" D'après le propriétaire, les animaux ont présenté, durant les premières quarante-huit heures, un état de demi-prostation avec colique et inappétence. Ces symptômes assez fugaces ont rapidement disparu et les bergers prétendent avoir trouver des vers dans les crottes expulsées dans les jours qui ont suivi.

"Le propriétaire a tout de même accusé une a mélioration notable de l'état général de ses animaux, avec une forte diminution de la mortalité.

"Les nécessités du service et l'éloignement de ce troupeau ne m'ont pas permis de contrốler moi-même les effets du traitement. Je me propose de le faire dès que possible, d'autant que ces effets sont particulièrement sensibles cette annẻe qui a été et sera si sévère pour nos troupeaux dans nos régions.

«Les 300 animaux traités à Mouillah, près de Djelfa, contenaient une plus forte proportion de malades. Je crois même pouvoir dire qu'ils constituaient le rebut des troupeaux de 'plusieurs membres d'une même famille; rebut restant constamment à proximité de la demeure du chef de famille et sous la surveillance directe des propriétaires.

“ Mouillah étant à proximité de D’jelfa, il m'a été plus facile de contrôler cette expérience.

"Comme pour le premier troupeau, quelques coliques fugaces avec inappétence ont attiré l'attention des éleveurs qui m'ont, en outre, signalé quelques émissions d'urines fortement colorées en rouge.

" J'ai eu l'occasion de revoir ce troupeau, le 23 Mai 1944. Sa physionomie a entièrement changé, il reste à peine $10 \%$ d'animaux souffreteux, tout le reste a l'œil vif et est en bon état d'embonpoint. Or, ce n'est pas le pacage, particulièrement pauvre ce printemps et dans la région 
considérée, qui peut suffire à expliquer cette résurreçtion. : Du reste, l'opinion des propriétaires est formelle: la mortalité a cęssé etole produit leur paraît très efficace. La strongylose pulmonaire, èlle aussi, semble avoir rétrocédé, ceci trouve une explication dans líextrême pulvérulence de lá phénothiazine : une certaine proportion du produit pénétrànt dans l'arbre respiratoire où, peut-être, un certain nombre de parasites sont alors détruits.

"Conclusions : La phénothiazine semble devoir donner d'excellents résultats dans le traitement des strongyloses ovines et caprines. Son administration, sous forme de poudre et à l'aide d'une cuillère à soupe, parait relativement simple, sûre et aisée. Son emploi mérite d'être vulgarisé le plus tôt possible, notamment cette année où l'enzootie risque de prendre une nouvelle ampleur, en raison des circonstances climatériques particulièrement défavorables dans tout le pays du mouton.:

«Cependant, il serait sonhaitable de pouvoir traiter d'une façon suivie un ou plusieurs troupeaux; de façon à pouvoir tirer des conclusions pratiques irrécusables. Comme pour l'emploi de la poudre de Theiler et en raison de l'évolution des strongles, un traitement prophylactique périodique s'impose. Sans doute, lá fréquence: des traitements successifs peut-elle être diminuée en raison de l'efficacité plus grande de la phénothiazine qui nous paraît constituer un traitement de choc remarquable? De toute façon, il me paraît souhaitable que le traitement d'entretien succède à ce traitement de choc.

« Dans nos régions, nous n'en sommes malheureusement pas encore là et c'est en cela que la phénothiazine peut nous rendre de grands services. Les éleveurs risquent d'être favorablement impressionnés par l'effet immédiat: arrêt de la mortalité, amélioration de l'état de leurs animaux. Quant au traitement d'entretien, ce n'est que plus tard que nous pourrons essayer de le faire entrer dans leurs mœurs. ")

Poưr -administrer la phénothiazine, le mode opératoire suivant fut préconisé :

Un aide debout prend le mouton entre ses jambes, les genoux maintenant la cage thoracique en arrière des épaules afin d'éviter le recul de l'animal. Il prend la tête entre ses mains en l'allongeant sur le cou, passe les pouces dans les espaces interdentaires infẻrieur et supérieur en prenant soin de ne pas retourner vers l'intérieur les commissures des lèvres. Il effectue alors une double traction de sens opposée de façon à ouvrir la bouche et la maintenir grande ouverte durant le temps d'administration du médicament. L'opérateur introduit la cuillère bien pleine et lui fait subir une rotation sur elle-même d'un quart de tour pour permettre au produit de glisser dans la bouche. Dès que la cuillère est retirée, l'aide referme les mâchoires et les maintient serrées, jusqu'à ce que l'animal fasse un ou deux mouvements de déglutition. 
Généralement, l'absorption est facile, quelquefois un effort dé toux rejette une partie de la poudre à l'extérieur : la quántité ingérée semble, la plupart du temps, suffisamment importante.

Lorsque aide et opérateur sont familiarisés ávec la technique, la cadence d'administration est assez rapide. D'autre part, le peu de complexité du mode opératoire et de l'instrumentation permet à tout éleveur, un peu consciencieux, de pratiquer lui-même cette administration.

La Propagande et la Statistique. - Unè première partie du problème se trouvait ainsi résolue. Restait à organiser la propagande.

Pour ce faire, l'Inspection du Service de l'Élevage allait utiliser deux moyens : la progagande verbale assurée par les vétérinaires des circonscriptions moutonnières, les administrateurs des communes mixtes, la radio en langue arabe, les éleveurs européens; la propagande écrite assurée grâce à la-diffusion d'affiches placardées dans tous les mảrchés, les douars, les communes mixtes et chez les chefs indigènès.

Cette propagande serait renforcée par des distributions de primes diplômes, médailles, prix en espèces - à l'occasion des concours annuels de moutons, attribuées aux pasteurs qui auraient traité leurs troupeaux contre les maladies parasitaires, les strongyloses en particulier.

Malgré la mobilisation de nombreux vétérinaires, les inspecteùrs maintenus dans les circonscriptions moutonnières entreprirent, dès réception des instructions nécessaires, leurs démarches auprès des grands pasteurs et commencèrent, soit à l'occasion d'une vaccination, soit à l'occasion d'une visite de marché, les longues palabres obligatoires en pays musulman avànt tout accord définitif.'

En même temps, l'Inspection Centrale de l'Élevage organisait son dépôt de médicaments, ses expéditions et l'inévitable comptabilité qui les accompagne, faisait frapper des médailles, mettait à l'impression des affiches. Tout cela, rappelons-le, en pleine guerre, au milieu des restrictions de toutes sortes : restriction de métal, restriction de papier et, aussi, pénurie de personnel, difficulté des transports, irrégularité des communications, etc...

Les premières distributions massives de phénothiazine aux moutons commencèrent en Juin 1944: En Décembre de la même année, $1.361 \mathrm{kgs}$ déce produit avaient été livrés aux pasteurs musulmans, par l'intermédiaire des vétérinaires du service de l'Élevage, ce qui représentait le traitement de 45.000 moutons environ. En 1945, les expéditions deviennent plüs importantes. En un seul mois (Juillet 1945); $1.836 \mathrm{kgs}$ de phénothiażine partent du dépôt de l'Élevage à destination du sud. Au 31 Décembre $1945,5.500 \mathrm{kgs}$ ont été administrés, et 183.000 ovins traités.

Peu à peu, la propagande effectuée dans tous les points du territoire 
porte ses fruits. La cadence des distributions de phénothiazine se fait à un rythme régulièrement accéléré et l'on peut dire que les résultats obtenus dans la lutte entreprise contre les strongyloses gastro-intestinales sont d'abord l'œuvre des vétérinaires affectés aux circonscriptions moutonnières, auxquels il a fallu beaucoup de patience et de force persuasive pour parvenir à ébranler l'apathie ou la méfiance profonde du monde pastoral algérien.

Le Contrôle scientifique. - Nous avons signalé plus haut l'absence d'une nomenclature complète de la faune parasitaire du cheptel ovin, en Algérie, - des nématodes en particulier.

Grâce aux crédits mis à sa disposition, le Service de l'Élevage put recruter, au titre des missions scientifiques, deux Assistantes qui, détachées de la Station Expérimentale d'Élevage d'Algérie - Centre de Recherches vétérinaires et zootechniques - au Laboratoire de Biologie générale et appliquée de la Faculté des Sciences, ont amorcé cette importante et longue étude systématique.

Les premières espèces dont la présence certaine a été reconnue dans les ovins d'Algérie a été publiée dans cette Revue (1947, n 1, p. 72). 